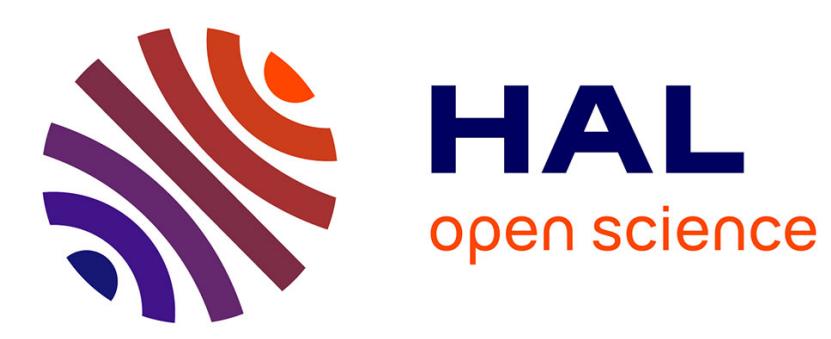

\title{
Asymptotic expressions describing radiative heat transfer between polar materials from the far-field regime to the nanoscale regime
}

\author{
Emmanuel Rousseau, Marine Laroche, Jean-Jacques Greffet
}

\section{To cite this version:}

Emmanuel Rousseau, Marine Laroche, Jean-Jacques Greffet. Asymptotic expressions describing radiative heat transfer between polar materials from the far-field regime to the nanoscale regime. Journal of Applied Physics, 2012, 111, pp.014311. 10.1063/1.3672809 . hal-00743615

\section{HAL Id: hal-00743615 \\ https://hal.science/hal-00743615}

Submitted on 19 Oct 2012

HAL is a multi-disciplinary open access archive for the deposit and dissemination of scientific research documents, whether they are published or not. The documents may come from teaching and research institutions in France or abroad, or from public or private research centers.
L'archive ouverte pluridisciplinaire HAL, est destinée au dépôt et à la diffusion de documents scientifiques de niveau recherche, publiés ou non, émanant des établissements d'enseignement et de recherche français ou étrangers, des laboratoires publics ou privés. 


\title{
Asymptotic expressions describing radiative heat transfer between polar materials from the far-field regime to the nanoscale regime
}

\author{
Emmanuel Rousseau, ${ }^{1}$ Marine Laroche, ${ }^{1}$ and Jean-Jacques Greffet ${ }^{1}$ \\ Laboratoire Charles Fabry, Institut d'Optique \\ CNRS, Univ Paris-sud \\ Campus Polytechnique RD 128 \\ 91127 Palaiseau cedex, France
}

\begin{abstract}
Heat transfer between two plates of polar materials at nanoscale distance is known to be enhanced by several orders of magnitude as compared with its far-field value. In this article, we derive accurate analytical expressions to quantitatively predict heat fluxes in the near-field. These analytical expressions reveal the physical mechanisms responsible for the enhancement. For two dielectric polar materials and for gaps smaller than 75 $\mathrm{nm}$ at the room temperature the heat transfer is dominated by the surface phonon polariton contribution. Between $75 \mathrm{~nm}$ and $500 \mathrm{~nm}$, the enhancement is mostly due to frustrated total internal reflection. The paper reports accurate analytical expressions for both contributions. Our analytical results highlight two differences between radiation flux at the nanoscale and in the far field: i) the heat flux spectrum depends on the gap distance, ii) the temperature dependence of the heat transfer coefficient deviates strongly from the $T^{3}$ law valid for grey bodies in the far-field.
\end{abstract}

\section{INTRODUCTION}

The heat flux between two bodies in a vacuum is only due to radiative heat transfer. This transfer can be enhanced by many orders of magnitude when the distance separating the bodies becomes smaller than the thermal wavelength $\lambda_{T}=\frac{\hbar c}{k_{B} T}$ i.e. $7.6 \mu \mathrm{m}$ at room temperature ( $\hbar=h / 2 \pi$ is Planck constant, $k_{B}$ is Boltzmann constant, $c$ is the light velocity and $T$ is the temperature). This is due to energy tunneling mediated by evanescent waves whose wavevector component parallel to the interface is larger than $\omega / c$ where $\omega$ is the circular frequency. Cravalho et al. ${ }^{1}$ were the first to point out the role of the evanescent waves. However they considered only the case where evanescent waves in the gap result from Frustrated Total Internal Reflection (FTIR) of propagating waves in the material. They also restricted their work to the case of materials with a low imaginary part of the refractive index. Within the framework of the fluctuational electrodynamics ${ }^{2}$ their work has been extended ${ }^{3-11}$ to account for modes with parallel wave vectors larger than $n_{r} \omega / c$ where $n_{r}$ is the real part of the optical index. In particular, it has been shown ${ }^{8,12}$ that for materials supporting surface phonon polaritons the radiative heat transfer is enhanced by an order of magnitude. Recent experiments ${ }^{13-15}$ have taken advantage from this enhancement and a good agreement with the Polder-Van Hove theory ${ }^{3,8}$ has been reported ${ }^{15}$. These measurements were made possible because the heat flux increases as $1 / l^{2}$ in the case of two infinite planes separated by a vacuum gap $l$. Yet, in the experiment, a sphere-plane geometry is used so that different distance regimes are involved. In addition, there have been little attention devoted to the temperature dependence of the heat transfer.

Here, we report accurate analytical expressions for the heat transfer between two surfaces supporting surface phonon polaritons. By properly accounting for the detailed dispersion relation of the surface modes, we are able to significantly improve previously reported semiquantitative formulas ${ }^{11}$. Our approach highlights the fundamental role of the density of states and the transmission coefficient of each mode as pointed out in two recent papers ${ }^{16,17}$. This approach is not restricted to the surface phonon polariton contribution (quasi-static limit). We used it beyond the quasi-static limit and give asymptotic formulae which are valid for gap widths up to $500 \mathrm{~nm}$. Furthermore our analytical formulas do not depend on a specific model of the dielectric constant. They can be applied to a wide range of situations when a proper estimate of the heat transfer is required. Such expressions can be useful in order to control $^{17-19}$ heat fluxes at nanometric scales.

\section{DESCRIPTION AND MODELLING}

\section{A. Description of the system}

The system studied consists in two infinite media separated by a vacuum gap $l$ (see Fig. 1). Their temperatures are supposed to be uniform and noted $T$ and $T+\delta T$. We assume that the temperature difference is small so that we can linearize the flux and introduce a heat transfer coefficient $h_{t o t}(l, T)$ defined as:

$$
\varphi(l, T)=h_{t o t}(l, T) \delta T
$$

where $\varphi(l, T)$ is the radiative flux, $\mathrm{T}$ the temperature of the first plate, $T+\delta T$ the temperature of the second plate (see Fig. 1).

We will use silicon carbide $(\mathrm{SiC})$, amorphous silica $\left(\mathrm{SiO}_{2}\right)$ and alumina $\left(\mathrm{Al}_{2} \mathrm{O}_{3}\right)$ to illustrate our results. As previously seen, optical properties affect heat $\operatorname{transfer}^{8,20}$. Amorphous silica and alumina optical properties are taken from Ref. ${ }^{21}$. Since optical properties 


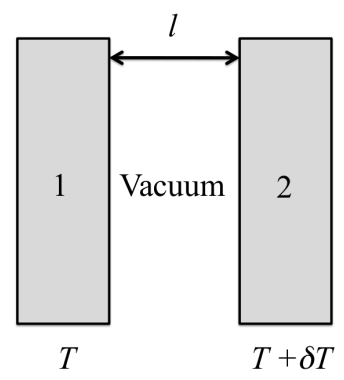

FIG. 1. Two semi-infinite half-spaces separated by a vacuum gap (distance $l$ ). Materials 1 and 2 can be identicals or differents.

variations with temperature are not known, an approximation is made here. We suppose that the optical properties remain the same in the range of temperature $[200 \mathrm{~K}, 600 \mathrm{~K}]$. On the contrary concerning silicon carbide we use the Lorentz model which describes with great accuracy the optical index ${ }^{21,22}$. Four parameters are needed to describe the optical properties: the dielectric function at high frequency $\varepsilon_{\infty}$, the longitudinal and the transverse optical-phonon frequency $\left(\omega_{L O}\right.$ and $\omega_{T O}$ respectively) and a damping factor $\Gamma$.

$$
\varepsilon(\omega)=\varepsilon_{\infty}\left(\frac{\omega_{L O}^{2}-\omega^{2}-i \Gamma \omega}{\omega_{T O}^{2}-\omega^{2}-i \Gamma \omega}\right)
$$

No approximations are done here concerning temperature variations since these four parameters have been measured ${ }^{22}$ at five different temperatures in the range [300-800K]. The high-frequency dielectric function $\varepsilon_{\infty}$ and the optical-phonon frequencies $\omega_{T O}$ and $\omega_{L O}$ change by less than $2 \%$ and will be taken to be constant. On the other hand, the damping factor increases linearly with temperature. The fit parameters are: $\Gamma(T)=$ $4.8329+0.0183(T-300) \mathrm{cm}^{-2}$. Note that this linear fit yields a value at $300 \mathrm{~K} \Gamma(300 \mathrm{~K})=4.8329 \mathrm{~cm}^{-2}$ that differs by $1.5 \%$ from the tabulated value $\left(4.76 \mathrm{~cm}^{-2}\right)$ in Ref. ${ }^{21}$.

\section{B. Theory}

Computing radiative heat transfer in the presence of evanescent waves requires a full electromagnetic approach ${ }^{3,5}$ in the framework of fluctuational electrodynamics ${ }^{4}$ as introduced by Rytov. The flux is obtained by calculating the Poynting vector across the gap separating the two bodies as shown in $\operatorname{refs}^{7,8,10,11}$ :

$$
h_{\text {tot }}(l, T)=\sum_{i=s, p} \int_{0}^{\infty} d \omega\left[h_{\text {prop }}^{i}(l, T, \omega)+h_{\text {evan }}^{i}(l, T, \omega)\right]
$$

where the sum over $i=s(T E), p(T M)$ accounts for the two polarizations. We have introduced the contribution of the propagating modes $(\kappa<\omega / c)$ :

$$
\begin{aligned}
& h_{\text {prop }}^{s, p}(l, T, \omega)=h^{0}(\omega, T) \times \\
& \int_{0}^{k_{0}} \frac{\kappa d \kappa}{k_{0}^{2}} \frac{\left(1-\left|r_{31}^{s, p}\right|^{2}\right)\left(1-\left|r_{32}^{s, p}\right|^{2}\right)}{\left|1-r_{31}^{s, p} r_{32}^{s, p} e^{-2 i \gamma l}\right|^{2}}
\end{aligned}
$$

and the contribution of the evanescent $\operatorname{modes}(\kappa>\omega / c)$ :

$$
\begin{aligned}
& h_{\text {evan }}^{s, p}(l, T, \omega)=h^{0}(\omega, T) \times \\
& \int_{k_{0}}^{\infty} \frac{\kappa d \kappa}{k_{0}^{2}} \frac{4 \operatorname{Im}\left(r_{31}^{s, p}\right) \operatorname{Im}\left(r_{32}^{s, p}\right)}{\mid 1-r_{31}^{s, p} r_{32}^{s, p} e^{-\left.2 \gamma^{\prime \prime l}\right|^{2}}} e^{-2 \gamma^{\prime \prime} l},
\end{aligned}
$$

where $h^{0}(\omega, T)$ is the derivative of the blackbody intensity:

$$
h^{0}(\omega, T)=\pi \frac{\partial L^{0}}{\partial T}(\omega, T)=\frac{1}{T} \frac{\hbar \omega}{k_{b} T} \frac{\hbar \omega^{3}}{4 \pi^{2} c^{2}} \frac{1}{\left[2 \sinh \left(\frac{\hbar \omega_{0}}{2 k_{b} T}\right)\right]^{2}} .
$$

$r_{31}^{s, p}$ and $r_{32}^{s, p}$ are the Fresnel coefficient of each interface (3 denotes the vacuum) and are given in the Appendix A. The Fresnel coefficients depend on the polarization $\mathrm{s}$ or $\mathrm{p}$ of the incident wave.

$\gamma^{\prime \prime}=\operatorname{Im}[\gamma]$ is the imaginary part of the z-component $\gamma=\sqrt{(\omega / c)^{2}-\kappa^{2}}$ of the wave vector in the vacuum gap whereas $\kappa$ is the component parallel to the interface. They satisfy the relation: $\kappa^{2}+\gamma^{2}=(\omega / c)^{2}$. We also define $k_{0}=\omega / c$.

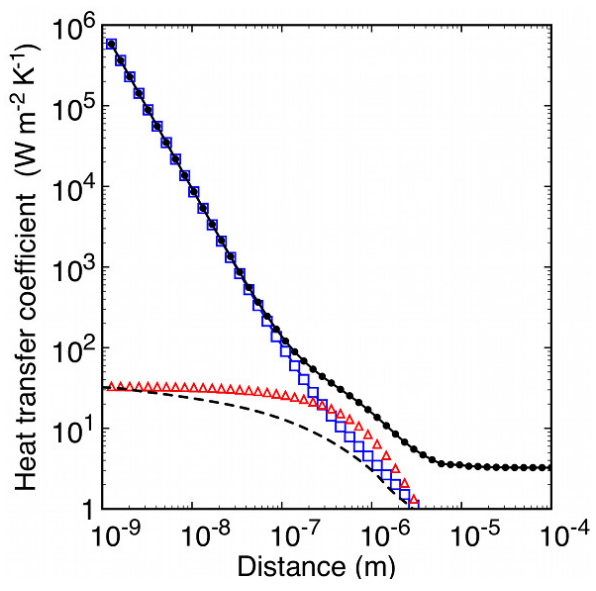

FIG. 2. Evolution of the heat transfer coefficient with distance for two infinite planes of SiC. Black dots curve: Total heat transfer coefficient $\left(h_{t o t}\right)$. Red triangles curve: Evanescent s-polarized contribution $\left(h_{\text {evan }}^{s}\right)$. Blue square curve: Evanescent p-polarized $\left(h_{\text {evan }}^{p}\right)$. Black dashed line: Contribution of p-polarized evanescent waves coming from frustrated total internal reflections. The average temperature is the room temperature $(\mathrm{T}=300 \mathrm{~K})$. Colors are available online.

The dependence of the heat transfer coefficient for two slabs of $\mathrm{SiC}$ as a function of the gap distance is shown in Fig. 2. It represents the total heat transfer coefficient, its 
evanescent p- and s-polarized component versus the gap distance $l$. We can distinguish three regimes: 1) For gaps smaller than $\lambda_{T} / 100$ (i.e. $75 \mathrm{~nm}$ at room temperature), the heat transfer is mainly dominated by the p-polarized evanescent waves contribution (blue squares curve in Fig. 2. 2)Between $75 \mathrm{~nm}$ and $500 \mathrm{~nm}$ there is an intermediate regime where both s- and p-polarized contribution have to be taken into account. 3) For a distance larger than $\lambda_{T}$ ( i.e. $7.6 \mu \mathrm{m}$ at room temperature), the heat transfer coefficient is independent of the distance when the propagating part of equation (3) dominates the heat transfer.

\section{ASYMPTOTIC EXPRESSIONS FOR EXTREME NEAR-FIELD REGIME}

In this section we focus on the regime where the ppolarization dominates the heat transfer i.e. for gaps smaller than $\lambda_{T} / 100$.

In this extreme near-field regime, the heat transfer coefficient reduces to:

$$
h(l, T) \simeq \int_{0}^{\infty} d \omega h_{e v a n}^{p}(l, T, \omega)
$$

\section{A. Closed-form expression of the heat transfer coefficient in the electrostatic limit}

We first turn to the calculation of the heat transfer coefficient in the electrostatic limit. In this limit surface modes contribution dominates the heat transfer ${ }^{8,16}$. We rewrite equation (5) with dimensionless units: $u=$ $\hbar \omega / k_{B} T, \tilde{\kappa}=\kappa / k_{0}, \tilde{\gamma}=k_{0} \sqrt{\tilde{\kappa}^{2}-1}, k_{T}=1 / \lambda_{T}:$

$$
h_{\text {evan }}^{p}(l, T)=\int_{0}^{\infty} d u h_{\text {evan }}^{p}(l, T, u)
$$

with

$$
\begin{aligned}
& h_{\text {evan }}^{p}(u, l, T)=\frac{3}{2 \pi^{3}} \frac{g_{0}}{\lambda_{T}^{2}} h^{0}(u) \times \\
& \int_{0}^{\infty} \tilde{\gamma} d \tilde{\gamma} \frac{4 \operatorname{Im}\left(r_{31}\right) \operatorname{Im}\left(r_{32}\right) e^{-2 k_{T} \tilde{\gamma} u l}}{\left|1-r_{31} r_{32} e^{-2 k_{T} \tilde{\gamma} u l}\right|^{2}}
\end{aligned}
$$

where $g_{0}=\pi^{2} k_{B}^{2} T / 3 h$ is the quantum of thermal conductance.

Note that Fresnel coefficients are now function of $u$ and $\tilde{\kappa}$ and $h^{0}(u)=u^{4} e^{u} /\left(e^{u}-1\right)^{2}$. One can note that for evanescent waves:

$$
\frac{\operatorname{Im}\left(r_{31} r_{32}\right)}{\left|1-r_{31} r_{32} e^{-2 k_{T} \tilde{\gamma} u l}\right|^{2}} e^{-2 k_{T} \tilde{\gamma} u l}=\operatorname{Im}\left[\frac{r_{31} r_{32} e^{-2 k_{T} \tilde{\gamma} u l}}{1-r_{31} r_{32} e^{-2 k_{T} \tilde{\gamma} u l}}\right]
$$

This allows to obtain an analytical form of (7) by removing the modulus function. In the electrostatic regime $(\tilde{\kappa} \gg 1)$, the Fresnel coefficients are independent of the parallel wave vector $\tilde{\kappa}$ : $\tilde{r}_{p}=\frac{\varepsilon(u)-1}{\varepsilon(u)+1}$. A final change of variable $X=2 \tilde{\gamma} k_{T} u l$ yields the surface-phonon polariton contribution noted $h_{s p p}$ :

$$
\begin{aligned}
& h_{s p p}(u, l, T)=\frac{3}{2 \pi^{3}} \frac{g_{0}}{l^{2}} \frac{h^{0}(u)}{u^{2}} \times \\
& \frac{\operatorname{Im}\left(\tilde{r}_{31}\right) \operatorname{Im}\left(\tilde{r}_{32}\right)}{\operatorname{Im}\left(\tilde{r}_{31} \tilde{r}_{32}\right)} \int_{0}^{\infty} X d X \operatorname{Im}\left[\frac{\tilde{r}_{31} \tilde{r}_{32} e^{-X}}{1-\tilde{r}_{31} \tilde{r}_{32} e^{-X}}\right]
\end{aligned}
$$

The distance dependence $\left(h_{s p p}(u, l, T) \propto 1 / l^{2}\right)$ is a consequence of the electrostatic limit and equation (8) can be integrated with the help of the polylogarithm function of the second order ${ }^{23}$ which takes into account the dispersion relation of the two infinite planes geometry:

$$
\begin{aligned}
& h_{s p p}(u, l, T)=\frac{3}{2 \pi^{3}} \frac{g_{0}}{l^{2}} \frac{h^{0}(u)}{u^{2}} \times \\
& \frac{\operatorname{Im}\left(\tilde{r}_{31}\right) \operatorname{Im}\left(\tilde{r}_{32}\right)}{\operatorname{Im}\left(\tilde{r}_{31} \tilde{r}_{32}\right)} \operatorname{Im}\left[\operatorname{Li} i_{2}\left(\tilde{r}_{31} \tilde{r}_{32}\right)\right]
\end{aligned}
$$
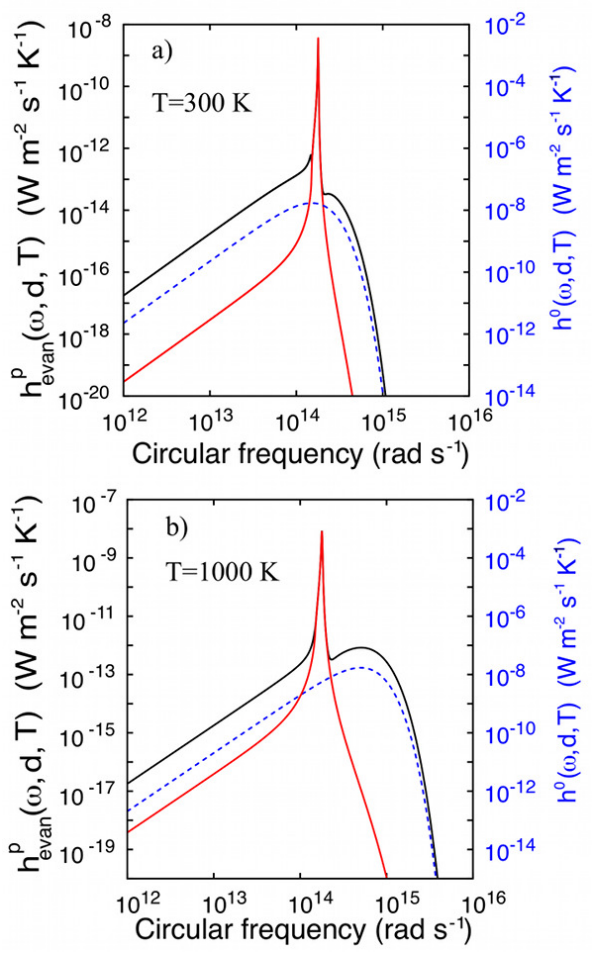

FIG. 3. Evolution of the the p-polarization of monochromatic heat transfer coefficient for two infinite planes of $\mathrm{SiC}$ at two different temperatures $\mathrm{T}=300 \mathrm{~K}(\mathrm{a})$ and $\mathrm{T}=1000 \mathrm{~K}(\mathrm{~b})$. The gap is $l=10 \mathrm{~nm}$. We represent only the p-polarization component. The black curve is the exact numerical result and the red line is the asymptotic expression (equation 9$)$. The dashed blue line is the derivative of the Planck function $h^{0}(\omega, T)$. 
No assumptions have been made on the form of the Fresnel coefficients except that they are taken in the electrostatic limit where they are independent of the parallel wave vector. This result has already been obtained in the case of doped silicon ${ }^{24}$ which supports surface-plasmon polariton in the infrared. In this paper we go further by performing the integration over the reduce frequency $u$ and we obtain an analytical formula (eq. (12)). Furthermore the method is generalized and we obtain then an equation that gives the near-field radiative heat transfer between two infinite planes without assuming any model for the dielectric constant (eq. (13)).

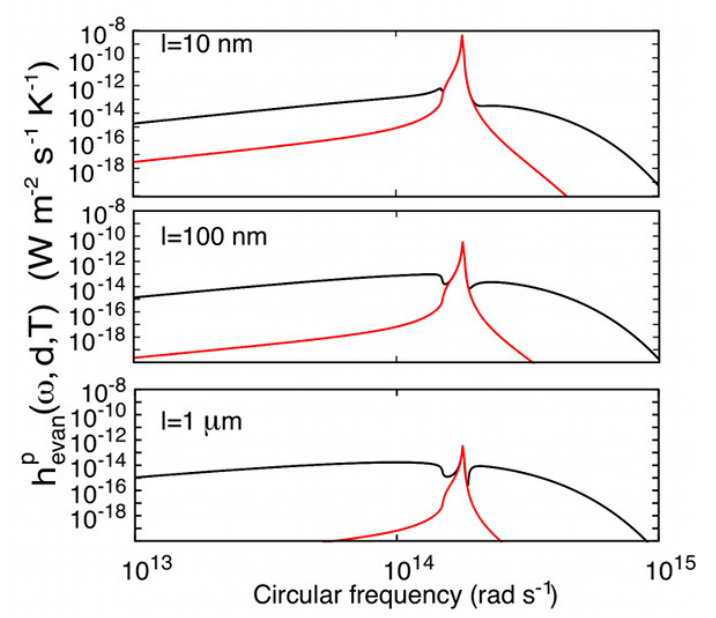

FIG. 4. Evolution of the p-polarization of the monochromatic heat transfer coefficient for two infinite planes of $\mathrm{SiC}$ for three different distances $l=1 \mu \mathrm{m}, l=100 \mathrm{~nm}, l=10 \mathrm{~nm}$. We represent only the p-polarization component. The black curve is the exact numerical result and the red line is the surfacephonon polariton contribution (Eq. 9). The temperature is $T=300 K$.

We now compare the closed-form expression $h_{s p p}(\omega, l, T)$ (Eq. 9) and a numerical integration of the p-polarisation heat transfer coefficient $h_{\text {evan }}^{p}(\omega, l, T)$ as a function of the frequency $\omega$.

The comparison is done for two temperatures in Fig. 3 and three distances in Fig. 4. The black curve is a numerical integration of Eq. (4). It describes only the contribution of the p-polarization. The red curves are the asymptotic expressions (Eq. 9) and the blue dashed curves are the derivative of the Planck function $h^{0}(\omega, T)$. The surface mode contribution $h_{s p p}(\omega, l, T)$ describes correctly the peak for a large range of distances $(l \leq 1 \mu \mathrm{m})$ and temperatures $(T \leq 1500 K)$.

For distances smaller than $5 \mathrm{~nm}$, the heat transfer is completely dominated by the surface mode which contributes for more than $99.5 \%$ to the total heat transfer at $300 \mathrm{~K}$. On the contrary at $100 \mathrm{~nm}$ only $48 \%$ of the total flux is due to the surface-phonon polariton coupling. Actually, the closed-form expression fails to describe the total curve and underestimates the contribution of the low and high frequency modes. These modes follow the
Planck function as it can be seen on Fig. 3-a and 3-b by considering the black $\left(h_{\text {evan }}^{p}(\omega, l, T)\right)$ and dashed blue curves $\left(h^{0}(\omega, T)\right)$ at low and high frequencies.

Fig. 4 shows that the spectrum of the heat flux depends on the distance. This is a specific near-field feature as in a vacuum, the far-field radiative heat flux spectrum is always independent on the distance. Yet, mainly the peak due to the surface phonon polariton depends on the distance and varies as $l^{-2}$. The remaining contributions have a spectrum which is weakly dependent on the distance $l$. Their contribution is analyzed in section IV.

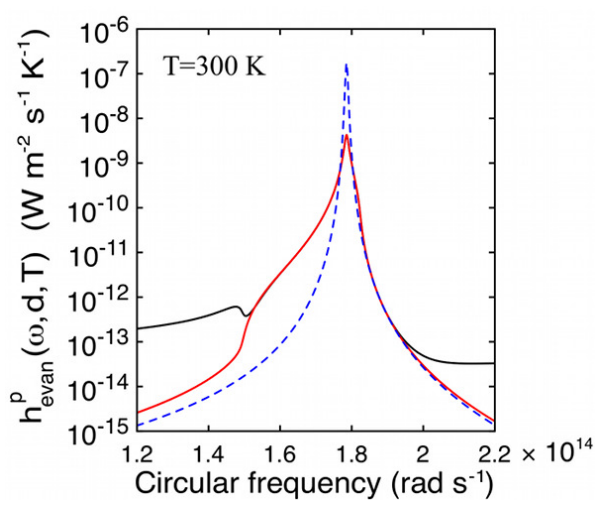

FIG. 5. Evolution of the p-polarization of the monochromatic heat transfer coefficient at $\mathrm{T}=300 \mathrm{~K}$ for two infinite planes of $\mathrm{SiC}$. The gap is $l=10 \mathrm{~nm}$. Black curve: p-polarization contribution to the monochromatic heat transfer coefficient obtained by a numerical integration of $h_{\text {evan }}^{p}(\omega, d, T)$ (Eq.4). This is the exact result. Red curve: Contribution to the surface mode $h_{s p p}(\omega, d, T)$. The dashed blue line is a rough estimate from Ref. ${ }^{5,8}$.

We now focus on the peak seen in figures 3 and 4 . In Fig. 5, we compare two different asymptotic formulae. The dashed blue line is a rough asymptotic estimate of the heat transfer coefficient derived in Ref. ${ }^{5,8}$. While it reproduces correctly the distance dependance $\left(h \propto 1 / l^{2}\right)$, this expression does not yield the correct amplitude and the correct width of the heat transfer coefficient. This is due to the fact that the dispersion relation of SPP in a gap had not been taken into account and that the transmission factor is incorrectly assumed to be 1 independently of the parallel wave-vector $\kappa$. Finally, let us emphasize that the closed-form expression Eq. (9) is valid in a plane-plane geometry with similar or different polar materials.

\section{B. Dependence of the near-field conductance on the temperature}

For a grey body, (i.e. a body with an emissivity $\epsilon$ independent on the the frequency), the far-field heat flux coefficient varies as $4 \epsilon \sigma T^{3}$. In this section, we investigate the dependence of the near-field heat transfer coefficient versus temperature. As we have just shown before, the heat flux spectrum is dramatically modified in 

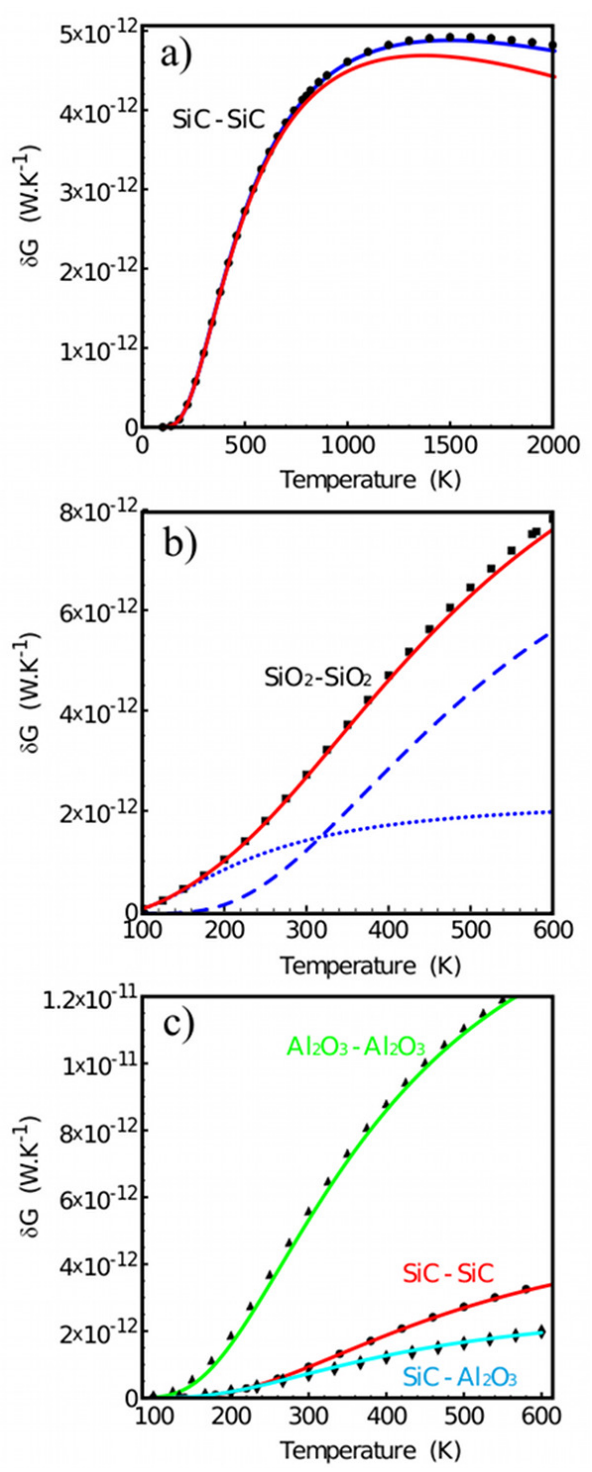

FIG. 6. Evolution of $\delta G$ with temperature for different materials. In all curves, symbols are extracted from the exact numerical integration of Eq. (4). Materials are indicated near the curves (a) The blue line is a numerical integration of the asymptotic expression Eq. (11). The red curve is the analytical formula Eq. (12) b) The red curve is the analytical expression Eq. (13) whereas the dotted and the dashed blue curves are the contribution of each resonance frequency $\left(\omega_{\mathrm{SiO}_{2}-\mathrm{SiO}_{2}}^{1}=9.2610^{13} \mathrm{rad} / \mathrm{s}\right.$ and $\omega_{\mathrm{SiO}_{2}-\mathrm{SiO}_{2}}^{2}=$ $2.1710^{14} \mathrm{rad} / \mathrm{s}$ respectively.). (c) Plain lines come from Eq. (13).

the short-distance regime and becomes quasi monochromatic. Hence, the temperature dependence which results from the integral over all frequencies is expected to be significantly modified in this particular regime. To this aim, we first define $\delta G(T)$ as:

$$
h_{s p p}(l, T)=\frac{\delta G(T)}{l^{2}}
$$

with

$$
\begin{aligned}
& \delta G(T)=\frac{3}{2 \pi^{3}} g_{0} \times \\
& \int_{0}^{\infty} d u \frac{h^{0}(u)}{u^{2}} \frac{\operatorname{Im}\left(\tilde{r}_{31}\right) \operatorname{Im}\left(\tilde{r}_{32}\right)}{\operatorname{Im}\left(\tilde{r}_{31} \tilde{r}_{32}\right)} \operatorname{Im}\left[L i_{2}\left(\tilde{r}_{31} \tilde{r}_{32}\right)\right]
\end{aligned}
$$

Our task is now to perform the integration over all frequencies in order to derive the heat transfer coefficient as a function of temperature. This is done in Appendix $\mathrm{B}$ in the case of two $\mathrm{SiC}$ planes. We find that that the contribution of the surface modes can be cast in the form :

$$
\begin{aligned}
& \delta G(T)=-\frac{3}{2 \pi^{2}} g_{0} \times \\
& \frac{\Gamma_{u}}{4} u_{s p p}^{2} \frac{e^{u_{s p p}}}{\left(e^{u_{s p p}}-1\right)^{2}} \operatorname{Re}\left[\operatorname{Li}_{2}\left(\tilde{r}^{2}\left(u_{s p p}\right)\right]\right.
\end{aligned}
$$

Where $\Gamma_{u}=\hbar \Gamma / k_{B} T$ is proportional to the material losses $\Gamma$ and $u_{s p p}$ is the single-interface surface-phonon polariton resonance. $\delta G(T)$ is shown in Fig. 6-a). First we compare the numerical computation of Eq. (4) (black dots) and a numerical integration of Eq. (11) (blue line) in order to test the accuracy of the electrostatic limit. The two curves perfectly fit for temperatures lower than $1500 \mathrm{~K}$. A small discrepancy appears at high temperature. It can be attributed to the failure of the electrostatic limit. High frequency modes are activated by the Planck function and the condition $\kappa \geq \omega / c$ can be no longer satisfied. Moreover it is clearly seen that $\delta G(T)$ presents a maximum for $T \simeq 1500 \mathrm{~K}$. This temperature behaviour differs drastically from the blackbody. For a blackbody, the flux always increases with increasing $T$ because the number of modes thermally activated increases. In the electrostatic regime the number of modes is fixed by the vacuum gap between the two slabs.

Now we compare the analytic result Eq. (12) (red curve) and the black dots which are a numerical integration of Eq. (4). Although the difference is $7 \%$ at 2000 $\mathrm{K}$ the accuracy is better than $2 \%$ for temperatures lower than $1000 \mathrm{~K}$. This discrepancy comes mainly from the approximation $u_{B} \simeq u_{s p p}$ meaning that the surface-mode frequency is independent of losses which is no more correct when the temperature increases. Another source of errors at high temperatures comes from the fact that we have neglected the contribution of the poles of the Planck function. Nevertheless Eq. (12) is simple and gives an accurate result for useful temperatures.

We now study the general case of two different materials. We do not assume any model for the dielectric constants. They can be given by experimental data. As shown in the Appendix B the pre-factor $\frac{\operatorname{Im}\left(\tilde{r}_{31}\right) \operatorname{Im}\left(\tilde{r}_{32}\right)}{\operatorname{Im}\left(\tilde{r}_{31} \tilde{r}_{32}\right)}=$ $\frac{1}{f(u)}$ plays an important role since it determines the poles of Eq. (11) (i.e. resonance frequencies) and the magnitude of the heat transfer. The previous study with two $\mathrm{SiC}$ half-spaces shows that: i) only poles of the pre-factor 
contribute to the heat transfer, poles of the fresnel coefficients do not, and, ii) theses poles are simples even in the case of similar materials. Such remarks enable us to integrate Eq. (11) in the general case of different materials described by experimental data. We find:

$$
\begin{aligned}
& \delta G(T)=\frac{3}{2 \pi^{2}} g_{0} \times \\
& \sum_{u_{i}} \frac{1}{f^{\prime}\left(u_{i}\right)} u_{i}^{2} \frac{e^{u_{i}}}{\left(e^{u_{i}}-1\right)^{2}} \operatorname{Re}\left[\operatorname{Li}_{2}\left(\tilde{r_{1}}\left(u_{i}\right) \tilde{r_{2}}\left(u_{i}\right)\right)\right]
\end{aligned}
$$

Where $u_{i}$ are zeros of $f(u)$ which give a negative value of the derivative $f^{\prime}\left(u_{i}\right)<0$. Theses frequencies are the coupled-mode resonances and give a positive contribution to the heat transfer. The other solutions $f\left(u_{j}\right)=0$ which lead to a positive value of the derivative $f^{\prime}\left(u_{j}\right)>0$ give a small negative contribution that is neglected. More details can be found the Appendix B.

Equation (13) and $f(u)$ are a key result of this paper since they give the first correct and general analytic closed-form expression for the heat transfer in the electrostatic regime. From $f(u)$ and the optical data, resonance frequencies and magnitude of the heat transfer coefficient can be obtained. Such equations can be used to optimize the heat transfer as it has been done in Ref. ${ }^{18,19}$.

The heat transfer between two slabs of silica is shown in Fig. 6-b). The squares curve is obtained from the exact numerical computation. The optical properties are taken from Ref. ${ }^{21}$. This material is interesting since its optical data exhibits two surface-phonon polariton resonances. Indeed two solutions satisfied $f\left(u_{i}\right)=0$ and $f^{\prime}\left(u_{i}\right)<0$. The corresponding frequency resonances are $\omega_{\mathrm{SiO}_{2}-\mathrm{SiO}_{2}}^{1}=9.2610^{13} \mathrm{rad} / \mathrm{s}$ and $\omega_{\mathrm{SiO}_{2}-\mathrm{SiO}_{2}}^{2}=$ $2.1710^{14} \mathrm{rad} / \mathrm{s}$. Injected into Eq. (13) they give respectively the dotted blue curve and the dashed blue curve. The contribution of the first coupled mode dominates the heat transfer at low temperature since only this mode is activated by the Planck function. When increasing the temperature the second coupled-mode contributes to the heat transfer. Eq. 13 perfectly reproduces $\delta G(T)$ variations with the temperature.

We now compare in Fig. 6-c) our closed-form expression for two different materials. $\delta G$, extracted from the exact numerical results, is plotted as diamonds in the case of a slab of $\mathrm{SiC}$ and a slab of alumina. As a comparison we also plot $\delta G$ for two slabs of $\mathrm{SiC}$ (circles) and two slabs of alumina (triangles). Plain curves are Eq. 13. One finds only one solution for the $\mathrm{SiC}-\mathrm{Al}_{2} \mathrm{O}_{3}$ geometry satisfying $f\left(u_{i}\right)=0$ and $f^{\prime}\left(u_{i}\right)<0$. This resonance frequency is $\omega_{\mathrm{SiC}-\mathrm{Al}_{2} \mathrm{O}_{3}}=1.6910^{14} \mathrm{rad} / \mathrm{s}$. For the $\mathrm{Al}_{2} \mathrm{O}_{3}-\mathrm{Al}_{2} \mathrm{O}_{3}$ system the frequency resonance is $\omega_{\mathrm{Al}_{2} \mathrm{O}_{3}-\mathrm{Al}_{2} \mathrm{O}_{3}}=1.5510^{14} \mathrm{rad} / \mathrm{s}$ and $\omega_{\mathrm{SiC}-\mathrm{SiC}}=$ $1.7910^{14} \mathrm{rad} / \mathrm{s}$ for the $\mathrm{SiC}-\mathrm{SiC}$ geometry. One can remark that dissimilar materials reduce the heat transfer coefficient. In summary Eq. 13 perfectly captures the physics of the heat transfer in the electrostatic limits since it reproduces the exact numerical data for a wide range of temperature even in the case of dissimilar materials.

\section{ASYMPTOTIC EXPRESSIONS FOR THE INTERMEDIATE NEAR-FIELD REGIME.}

As already mentioned, at room temperature the surface-phonon polariton dominates the heat transfer for distances smaller than $75 \mathrm{~nm}$. In order to describe the heat transfer in a wider range of gap widths one has to include the other modes contribution. In this section, we analyse the contribution of the evanescent waves coming from frustrated total internal reflections and find an asymptotic expression describing their contribution. We restrict our study to the case of two slab made of the same materials. Our results will be illustrated with silicon carbide.

\section{A. Contribution of evanescent waves coming from frustrated total internal reflection.}

These modes are characterized by a parallel wave vector that satisfies: $k_{0} \leq \kappa \leq n_{r}(\omega) k_{0}$ where $n_{r}(\omega)$ is the real part of the refractive index and $k_{0}$ denotes $\omega / c$ which defines the Frustrated Total Internal Reflection (FTIR) range. Note that the refractive index depends on the circular frequency and that the previous condition excludes all frequencies giving $n_{r}(\omega)<1$. In the case of $\mathrm{SiC}$ the previous condition excludes the window $\left[\omega_{T O}, \omega_{L O}\right]$.

We plot in Fig.2 the contribution of frustrated total internal reflections in $\mathrm{p}$ (dashed black line) and spolarization (red triangles). Both curves saturate and reach the same value at small gaps. Note that the scomponent reaches its saturation value for gaps larger than the p-component which saturates at extremely small gaps.

\section{B. Closed-form expression beyond the electrostatic limit}

The contribution of frustrated total internal reflections in s-polarization $h_{f}^{s}(\omega, l, T)$ and in p-polarization $h_{f}^{p}(\omega, l, T)$ are calculated in Appendix C. We recall here only the asymptotic form of the closed-form expressions in the first order of $\left(\frac{k_{0} l}{2}\right)^{2}$ since they exhibit two different length scales explaining why the saturation is reached at different gaps depending on the polarization:

$$
\begin{aligned}
& h_{f}^{s}(\omega, l, T) \simeq h_{\text {sat }}(\omega, T) \times \\
& \left\{1+\left(\frac{k_{0} l}{2}\right)^{2}\left(\varepsilon_{r}-1\right) \ln \left[\left(\frac{k_{0} l}{2}\right)^{2}\left(\varepsilon_{r}-1\right)\right]\right\}
\end{aligned}
$$

for the s-polarization and: 

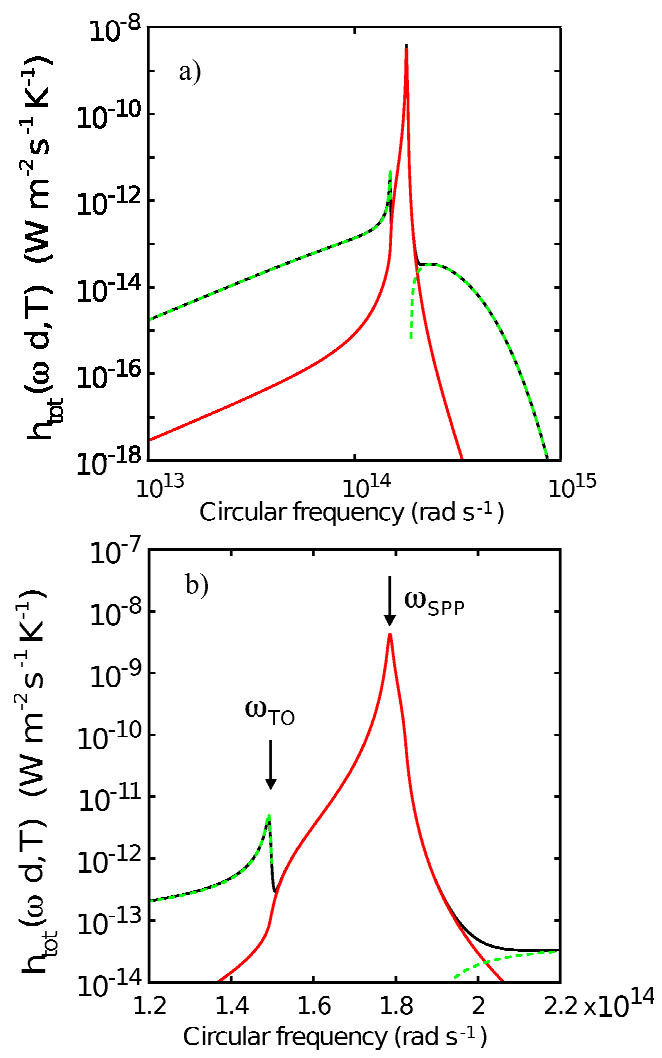

FIG. 7. Evolution of the total monochromatic heat transfer coefficient with circular frequency for two infinite planes of $\mathrm{SiC}$. The gap is $l=10 \mathrm{~nm}$ and the temperature is $T=300 \mathrm{~K}$. a) The black curve is the total monochromatic heat transfer coefficient (polarization $\mathrm{s}+\mathrm{p}$ ). It is obtained from a numerical integration of Eq. (3). The red line is the surface-mode contribution $h_{s p p}(\omega, d, T)$ (Eq. 9). The dashed green line is the contribution of the s- and p-polarization in the saturation regime $h_{f}^{\max }(\omega, T)$ given by Eq. (15). b) is a zoom in a narrow frequency range.

$$
\begin{aligned}
& h_{f}^{p}(\omega, l, T) \simeq h_{\text {sat }}(\omega, T) \times \\
& \left\{1+\left(\frac{k_{0} l}{2}\right)^{2}\left(\varepsilon_{r}-1\right) \varepsilon_{r}^{2} \ln \left[\left(\frac{k_{0} l}{2}\right)^{2}\left(\varepsilon_{r}-1\right) \varepsilon_{r}^{2}\right]\right\}
\end{aligned}
$$

for the p-polarization. In previous formulae $\varepsilon_{r}$ is the real part of the dielectric function. Validity ranges of these asymptotic expressions are given in the Appendix C.

For nearly touching slabs $l \simeq 0$ the same value is reached for both polarizations:

$$
h_{\text {sat }}(\omega, T)=h^{0}(\omega) \frac{n_{r}(\omega)^{2}-1}{2}
$$

The maximal heat transfer due to evanescent waves coming from frustrated total internal reflection is then given by:

$$
h_{f}^{\max }(\omega, T)=h^{0}(\omega)\left(n_{r}(\omega)^{2}-1\right)
$$

This limit has been previously obtained by Cravalho et $a l^{1}$ in the case of materials whose optical index is frequency independent and have a low imaginary part. Here we show that this simple formula giving the maximum heat flux due to frustrated total internal reflections can be extended to both s- and p-polarizations and for any materials supporting or not surface phonon polariton.

For a $10 \mathrm{~nm}$ gap, we plot in Fig.7-a the monochromatic total heat transfer coefficient (polarization $\mathrm{s}+\mathrm{p}$ ) $h_{\text {tot }}(\omega, d, T)$ (Eq. 4) obtained from exact numerical results versus the frequency. We also plot the surface mode contribution $h_{s p p}(\omega, d, T)$ (Eq. 9) and the contribution of frustrated total internal reflections $h_{f}^{s}(\omega, l, T)+$ $h_{f}^{p}(\omega, l, T)$. The sum of these three contributions now completely describes the exact numerical result. The shape and the peak are perfectly reproduced. We then focus on the peak seen in Fig.7-b. There are actually two peaks. One at $\omega_{\text {spp }}$ due to the surface-phonon polariton frequency and described by the surface mode contribution $h_{s p p}(\omega, T)$ as previously discussed. A small peak appears at $\omega_{T O}$. It results from the resonant behaviour of the dielectric constant when frequencies approach $\omega_{T O}$.

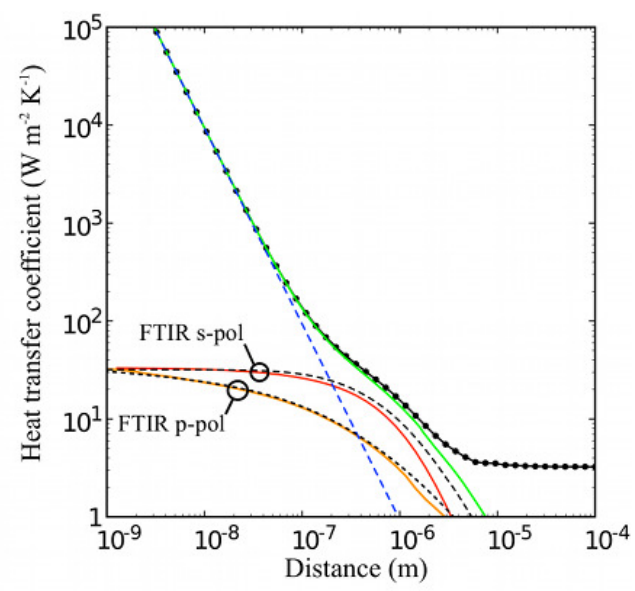

FIG. 8. Evolution of the total heat transfer coefficient with distance for two infinite planes of SiC. Black dots curve: Total heat transfer coefficient $h_{t o t}(l, T)$ (Eq. 3). Red plain curve: evanescent s-polarization contribution $h_{\text {evan }}^{s}(l, T)$ (Eq. 4). Orange plain curve: evanescent p-polarization contribution $h_{\text {evan }}^{p}(l, T)$ (Eq. 4) limited to the FTIR range. Next curves result from closed-form expressions. Blue dashed curve: contribution of the surface-phonon polariton $h_{s p p}^{p}(d, T)$ (Eq. 10). Black dashed curves are respectively FTIR s-pol $h_{f}^{s}(l, T)($ Eq. C2) for the FTIR contribution in s-polarization and FTIR p-pol $h_{f}^{p}(l, T)$ (Eq. C4) for the FTIR contribution in ppolarization The green curve is the approximate expression $h_{\text {approx }}(l, T)$ (Eq. 17).

We now focus on the distance dependence of the contribution of frustrated total internal reflections. To this 
aim we have to integrate over the circular frequencies:

$$
h_{f}^{s, p}(l, T)=\int_{\omega \notin\left[\omega_{T O}, \omega_{L O}\right]} d \omega h_{f}^{s, p}(\omega, l, T)
$$

The integration range is of course restricted to the FTIR range $\omega \notin\left[\omega_{T O}, \omega_{L O}\right]$ which makes the integration difficult to be performed analytically. Thus we numerically integrate it and compare with the exact results given by Eq. 4.

Black dashed curves in Fig. 8 are the result of the numerical integration of Eq. (16) with respectively Eq. (C2) (FTIR s-pol) and Eq. (C4) (FTIR p-pol) as the integrand for the s- or the p-polarization. The plain red and orange curves result from a numerical integration of Eq. 4. In this exact numerical integration we restrict the wave-vectors range to the window $\left[k_{0}, n_{r}(\omega) k_{0}\right]$ for the p-polarization (FTIR range) whereas we used the entire range $\left[k_{0},+\infty\right]$ for the s-polarization. Although the asymptotic expression $h_{f}^{s}(l, T)$ slightly overestimates the evanescent s-polarization contribution, the closed-form expression $h_{f}^{p}(l, T)$ perfectly reproduces the p-polarization contribution. Note that the distance dependence is perfectly reproduced in both case. Such a comparison prove that equations $(\mathrm{C} 2)$ and $(\mathrm{C} 4)$ capture the physics of the heat transfer beyond the electrostatic limit. As a consequence in s-polarization evanescent modes result only from frustrated total internal reflections which is expected for a non-magnetic material as $\mathrm{SiC}$.

One can note that for each polarization two regimes appear. A first one where the heat transfer coefficient varies slowly with the gap width and a second one for gaps larger than $l_{f}^{s, \star}$ in s-polarization or $l_{f}^{p, \star}$ in p-polarization where the heat transfer coefficient quickly decreases with the gap width $l$ (see Appendix $\mathrm{C}$ for definition of $l_{f}^{s, \star}$ and $\left.l_{f}^{p, \star}\right)$. Such behaviour can be understand with a mesoscopic point of view of the radiative heat transfer ${ }^{16,17}$. Our treatment of the contribution of evanescent waves coming from frustrated total internal reflection consists of fixing the number of modes by fixing the parallel wave vector in the range $k_{0} \leq \kappa \leq n_{r}(\omega) k_{0}$. Then we compute their transmission factor $A_{s, p}(\omega, \gamma, l, T)$. When gap widths are small compared to characteristic gap width $l \ll l_{f}^{s, \star}$ for s-polarization and $l \ll l_{f}^{p, \star}$ for p-polarization transmission factors are close to $A_{s, p} \simeq 1$. Each mode contributes then as the quantum of thermal conductance. Summing over all the allowed modes gives Eq.15. When the gap width increases the transmission factors $A_{s, p}$ decrease leading to a reduction of the thermal conductance whereas the number of contributing modes remains constant.

\section{VALIDITY RANGE.}

We now investigate the accuracy of the different asymptotic expressions and examine their validity range. We only consider the case of two silicon carbide slabs.
We define the approximate heat transfer coefficient as the sum of the surface-phonon polariton contribution and the contribution of frustrated total internal reflections in s- and p-polarization:

$$
h_{\text {approx }}(l, T)=h_{s p p}^{p}(l, T)+h_{f}^{s}(l, T)+h_{f}^{p}(l, T)
$$

where $h_{s p p}^{p}(l, T)$ is given by Eq. (10) and Eq. (13), $h_{f}^{s}(l, T)$ and $h_{f}^{p}(l, T)$ result from a numerical integration over the angular frequencies (Eq. (16)) of the closed-form expressions given by Eq. (C2) and Eq. (C4). The approximate coefficient $h_{\text {approx }}(l, T)$ (green curve) is compared with the exact numerical results (black dots) in the Fig. 8 at room temperature. With this procedure, the total heat transfer coefficient is well-described for distances up to $500 \mathrm{~nm}$ whereas the surface-phonon polariton $h_{s p p}^{p}(l, T)$ contribution (dashed blue curve in Fig. 8) can only describe the heat transfer coefficient for distance smaller than $75 \mathrm{~nm}$ at the room temperature. As an example, the relative difference between the exact numerical result (black dots) and the surface phonon contribution (dashed blue curve) is $48 \%$ at $d=100 \mathrm{~nm}$ whereas it is only $3 \%$ with the approximate $h_{\text {approx }}(l, T)$ (green curve).

Fig. 9 shows the relative difference between the total heat transfer $h_{t o t}(l, T)$ (Eq. 3) and the approximation $h_{\text {approx }}(l, T)$ (Eq. 17) for temperature varying between $[200-900 \mathrm{~K}]$ and gaps varying between $\left[10^{-9}, 10^{-5} \mathrm{~m}\right]$. The relative difference $(R)$ is in percent: $R=100 \times$ $\frac{\left|h_{\text {tot }}(l, T)-h_{\text {approx }}(l, T)\right|}{h_{\text {tot }}(l, T)}$.

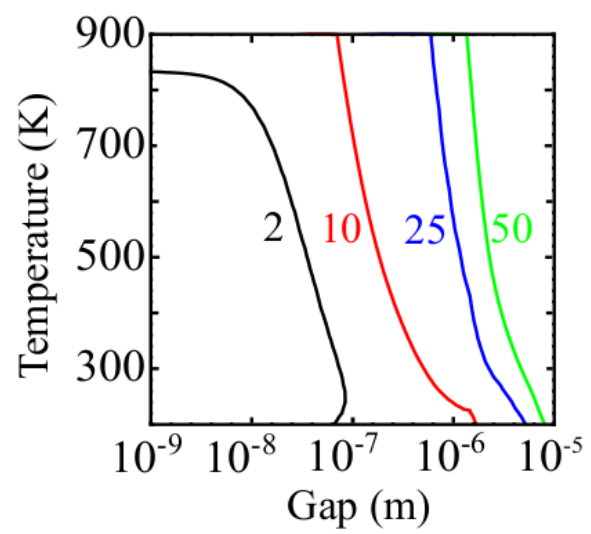

FIG. 9. Relative difference between the total heat transfer $h_{\text {tot }}(d, T)$ coefficient and the approximation $h_{\text {approx }}(d, T)$ in the case of two infinite planes of SiC. Numbers indicate the relative difference (in percent).

\section{CONCLUSION}

In this paper, we have shown that radiative heat transfer mainly results from the coupling of surface phononpolariton for distances shorter than $75 \mathrm{~nm}$. A careful 
analysis of their contribution allowed to derive a closedform expression of the heat transfer coefficient. It can be cast in the form $\varphi(l, T)=\frac{\delta G(T)}{l^{2}} S \delta T$. We have investigated the temperature dependence of $\delta G$ and we have found a behaviour markedly different from the blackbody radiation case. For distances larger than $75 \mathrm{~nm}$, other contributions must be included. We derived a closed-form expressions beyond the electrostatic limit in s- and p-polarization. This yields an analytical formula valid for distances up to $500 \mathrm{~nm}$ at room temperature. In summary, the results presented in this paper clarify the physical origin of the different contributions to the nanoscale heat transfer and provides closed-form expressions to compute quantitatively nanoscale heat transfer between polar materials.

The authors acknowledge the support of Agence $\mathrm{Na}$ tionale de la Recherche through Monaco projects (ANR06-NANO-062) and Leti-Carnot Institute.

\section{Appendix A: Fresnel coefficients}

The optical properties of the interface is included in the Fresnel reflection factors. Their explicit form is given below:

for the s-polarization:

$$
r_{31}^{s}=\frac{\gamma-\gamma_{1}}{\gamma+\gamma_{1}}
$$

with $\gamma=\sqrt{(\omega / c)^{2}-\kappa^{2}}$ the z-component of wave vector in the vacuum and $\gamma_{1}=\sqrt{\varepsilon_{1}(\omega / c)^{2}-\kappa^{2}}$ the $\mathrm{z}$ component of the wave vector in the material 1 .

and for the p-polarization:

$$
r_{31}^{p}=\frac{\varepsilon_{1} \gamma-\gamma_{1}}{\varepsilon_{1} \gamma+\gamma_{1}}
$$

\section{Appendix B: Temperature dependence of the heat transfer coefficient in the short distance regime}

In the electrostatic limit, the pre-factor $\frac{\operatorname{Im}\left(\tilde{r}_{31}\right) \operatorname{Im}\left(\tilde{r}_{32}\right)}{\operatorname{Im}\left(\tilde{r}_{31} \tilde{r}_{32}\right)}$ is $1 / f(u)$ with $f(u)=\frac{1}{2}\left(\frac{\left|\varepsilon_{1}\right|^{2}-1}{\operatorname{Im}\left[\varepsilon_{1}\right]}+\frac{\left|\varepsilon_{2}\right|^{2}-1}{\operatorname{Im}\left[\varepsilon_{2}\right]}\right)$. This function plays an important role since it determines the poles of Eq. (11) (i.e. resonance frequencies) and the magnitude of the heat transfer. In order to make our point clear we consider in this appendix the case of two slabs of silicon carbide. Then we integrate Eq. (11) in the general case of two different materials (see text).

For two $\mathrm{SiC}$ slabs, $f(u)$ is given by:

$$
f(u)=\frac{2}{\Gamma_{u}\left(u_{c h}^{2}-u_{s p p}^{2}\right)} \frac{\left(u^{2}-u_{c h}^{2}\right)\left(u^{2}-u_{s p p}^{2}\right)+\left(\Gamma_{u} u\right)^{2}}{u}
$$

where $u_{s p p}=\hbar \omega_{s p p} / k_{B} T$ with $\omega_{s p p}=\sqrt{\frac{\varepsilon_{\infty} \omega_{L O}^{2}+\omega_{T O}^{2}}{\epsilon_{\infty}+1}}$ the single-interface surface-phonon polariton resonant frequency, $u_{c h}=\hbar \omega_{c h} / k_{B} T$ with $\omega_{c h}=\sqrt{\frac{\varepsilon_{\infty} \omega_{L O}^{2}-\omega_{T O}^{2}}{\epsilon_{\infty}-1}}$ the Christiansen frequency $\omega_{c h}\left(\epsilon\left(\omega_{c h}\right)=0\right)$ and $\Gamma_{u}=$ $\hbar \Gamma / k_{B} T$.

$f(u)$ has 4 zeros: $\pm u_{A}, \pm u_{B}$ with:

$$
\begin{aligned}
u_{A}^{2} & =\frac{u_{s p p}^{2}+u_{c h}^{2}-\Gamma_{u}^{2}}{2}-\frac{\sqrt{\left(u_{s p p}^{2}+u_{c h}^{2}-\Gamma_{u}^{2}\right)^{2}-4 u_{s p p}^{2} u_{c h}^{2}}}{2} \\
& \simeq u_{c h}^{2}
\end{aligned}
$$

$$
\begin{aligned}
u_{B}^{2} & =\frac{u_{s p p}^{2}+u_{c h}^{2}-\Gamma_{u}^{2}}{2}+\frac{\sqrt{\left(u_{s p p}^{2}+u_{c h}^{2}-\Gamma_{u}^{2}\right)^{2}-4 u_{s p p}^{2} u_{c h}^{2}}}{2} \\
& \simeq u_{s p p}^{2}
\end{aligned}
$$

Note that those poles lie on the real axis on the contrary to the single-interface geometry where poles are complex numbers. We also emphasize that these poles coincide with the surface-phonon polariton frequency and the Christiansen frequency of the single-interface geometry only in the limit of low losses $\Gamma \ll \omega_{s p p}, \omega_{c h}$. This approximation is correct for temperatures lower than $1000 \mathrm{~K}$.

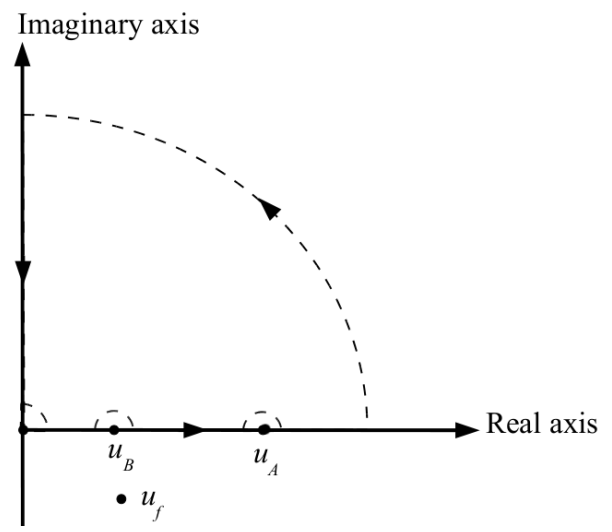

FIG. 10. Path of integration used to integrate eq. (11). $u_{A}$, $u_{B}$ and $u_{f}$ are poles of the integrand of eq. (11). $u_{A}$ and $u_{B}$ are poles of the pre-factor $\frac{\operatorname{Im}\left(\tilde{r}_{31}\right) \operatorname{Im}\left(\tilde{r}_{32}\right)}{\operatorname{Im}\left(\tilde{r}_{31} \tilde{r}_{32}\right)} \cdot u_{f}$ is a pole of the fresnel coefficients. The integration path excludes the origin, $u_{A}$ and $u_{B}$.

Now we will integrate Eq. (11) in the upper-positive part of the complex plane as plotted in Fig. 10. The path of integration is a quarter of circle whose radius extend to the infinity and exclude $u_{A}, u_{B}$ and the origin. The Fresnel coefficient's poles are $u_{f}= \pm \sqrt{u_{s p p}^{2}-\Gamma_{u}^{2} / 4}-$ $i \Gamma_{u} / 2$ and are excluded from this integration contour. The contribution of the pole $u_{A}$ (i.e. the Christiansen 
frequency) is small and negative and will be neglected. We also neglect the contribution of poles coming from the Planck function. We then obtain the following formula for the contribution of the coupled surface modes:

$$
\begin{aligned}
& \delta G(T)=-\frac{3}{2 \pi^{2}} g_{0} \times \\
& \frac{\Gamma_{u}}{4} u_{s p p}^{2} \frac{e^{u_{s p p}}}{\left(e^{u_{s p p}}-1\right)^{2}} \operatorname{Re}\left[\operatorname{Li}_{2}\left(\tilde{r}^{2}\left(u_{s p p}\right)\right]\right.
\end{aligned}
$$

Note that $\operatorname{Re}\left[\operatorname{Li}_{2}\left(\tilde{r}^{2}\left(u_{\text {spp }}\right)\right]\right.$ is negative so that $\delta G(T)$ is positive.

We now consider the general case of two different materials without assuming any model for dielectric constants. The integration path is the same so only poles of $\frac{1}{f(u)}$ contribute to the heat transfer. Integrating Eq. 11 gives:

$$
\begin{aligned}
& \delta G(T)=\frac{3}{2 \pi^{2}} g_{0} \times \\
& \sum_{u_{i}} \frac{1}{f^{\prime}\left(u_{i}\right)} u_{i}^{2} \frac{e^{u_{i}}}{\left(e^{u_{i}}-1\right)^{2}} \operatorname{Re}\left[\operatorname{Li}_{2}\left(\tilde{r_{1}}\left(u_{i}\right) \tilde{r_{2}}\left(u_{i}\right)\right)\right]
\end{aligned}
$$

Where $u_{i}$ are zeros of $f(u)$ which give a negative value of the derivative $f^{\prime}\left(u_{i}\right)<0$. This solution is equivalent to the coupled mode resonance $u_{B}$ in the previous case of two $\mathrm{SiC}$ slabs and give a positive contribution to the heat transfer. The other solution $f\left(u_{j}\right)=0$ which leads to a positive value of the derivative $f^{\prime}\left(u_{j}\right)>0$ is the equivalent of the Christiansen frequency $u_{A}$. This contribution is small and then neglected.

\section{Appendix C: Calculation of the contribution of evanescent waves coming from frustrated total internal reflection}

\section{1. s-polarization}

Let us estimate the value of the heat transfer coefficient due to frustrated total internal reflections. Following Pendry ${ }^{6}$, the integrand in Eq. 4 is bounded by:

$$
A_{s}=\frac{4 \operatorname{Im}\left(r_{s}\right)^{2} e^{-2 \gamma^{\prime \prime} d}}{\left|1-r_{s}^{2} e^{-2 \gamma^{\prime \prime} d}\right|^{2}} \leq 1
$$

The maximum value is reached in the following condition $^{6}$ :

$$
\left|r_{s}\right|^{2}=e^{2 \gamma^{\prime \prime} l}
$$

Evanescent modes coming from frustrated total internal reflections are characterized by $\left|r_{s}\right|^{2}=1$ so that condition $(\mathrm{C} 1)$ is satisfied in the asymptotic limit $l \rightarrow 0$. The heat transfer coefficient then saturates to a maximum value. In order to get the distance dependence we will develop $\left|r_{s}\right|^{2}$ around condition (C1) by writing $\left|r_{s}\right|^{2}=e^{2 \gamma l}+\left(1-e^{2 \gamma l}\right)$. One then gets:

$$
A_{s}(\omega, \gamma, l, T)=\frac{1}{1+\frac{\sinh \left[\gamma k_{0} l\right]^{2}}{I m\left[r_{s}\right]^{2}}} \simeq \frac{1}{1+\frac{\left(\gamma k_{0} l\right)^{2}}{I m\left[r_{s}\right]^{2}}}
$$

We recall here that we focus on the contribution of frustrated total internal reflections, i.e. modes characterized by $k_{0} \leq \kappa \leq n_{r}(\omega) k_{0}$. Such a condition implies that the real part of the optical index is greater than $1\left(n_{r}(\omega) \geq 1\right)$ and that its imaginary part is negligible $\left(n_{i}(\omega) / n_{r}(\omega) \ll 1\right)$. In the case of $\mathrm{SiC}$ these conditions excludes the range $\left[\omega_{T O}, \omega_{L O}\right]$. Through the following approximations the fresnel coefficient for evanescent modes coming from frustrated total internal reflections can be written as $r_{s}=\left(i|\gamma|-\sqrt{\varepsilon_{r}(\omega)-1-|\gamma|^{2}}\right) /(i|\gamma|+$ $\left.\sqrt{\varepsilon_{r}(\omega)-1-|\gamma|^{2}}\right)$ ) where $\varepsilon_{r}(\omega)$ is the real part of the dielectric constant. It follows that the imaginary part of the fresnel coefficient is given by $\operatorname{Im}\left[r_{s}\right]=$ $2 \frac{|\gamma|}{\sqrt{\varepsilon_{r}(\omega)-1}} \sqrt{1-\frac{|\gamma|^{2}}{\varepsilon_{r}(\omega)-1}}$.

Within these approximations we are now able to perform integration over the parallel waves vectors. The contribution of frustrated total internal reflections $h_{f}^{s}(\omega, l, T)$ to the monochromatic heat transfer coefficient is then given by:

$$
\begin{aligned}
& h_{f}^{s}(\omega, l, T)=h^{0}(\omega) \int_{0}^{k_{0} \sqrt{n_{r}(\omega)^{2}-1}} \frac{\gamma d \gamma}{k_{0}^{2}} A_{s}(\omega, \gamma, l, T) \\
& h_{f}^{s}(\omega, l, T)=h^{0}(\omega) \frac{n_{r}^{2}-1}{2} \times \\
& \left\{1+\left(\frac{k_{0} l}{2}\right)^{2} \frac{\left(\varepsilon_{r}-1\right)^{2}}{n_{r}^{2}-1} \ln \left[1-\frac{\frac{n_{r}^{2}-1}{\varepsilon_{r}-1}}{1+\left(\frac{k_{0} l}{2}\right)^{2}\left(\varepsilon_{r}-1\right)}\right]\right\}
\end{aligned}
$$

This is the general solution. In the case of two slabs of $\mathrm{SiC}$, the previous equation can be simplify because $\frac{\left(\varepsilon_{r}-1\right)}{n_{r}^{2}-1} \simeq 1$ all over the frequency range allowed by the requirement $k_{0} \leq \kappa \leq n_{r}(\omega) k_{0}$. In the first order of $\left(\frac{k_{0} l}{2}\right)^{2}\left(\varepsilon_{r}-1\right)$ the closed-form expression simplifies as:

$$
\begin{aligned}
& h_{f}^{s}(\omega, l, T) \simeq h^{0}(\omega) \frac{n_{r}^{2}-1}{2} \times \\
& \left\{1+\left(\frac{k_{0} l}{2}\right)^{2}\left(\varepsilon_{r}-1\right) \ln \left[\left(\frac{k_{0} l}{2}\right)^{2}\left(\varepsilon_{r}-1\right)\right]\right\}
\end{aligned}
$$

The heat transfer coefficient reaches its saturation value when the second term in the bracket becomes negligible i.e. when gap widths $l$ are smaller than $l_{f}^{s, \star}$ $l \ll l_{f}^{s, \star}=\frac{\lambda_{T}}{\pi} \frac{1}{\sqrt{\varepsilon_{r}\left(\omega_{T}\right)-1}}$ where $\lambda_{T}$ is the thermal wavelength and $\omega_{T}=\frac{k_{B} T}{\hbar}$ is the thermal circular frequency. At the room temperature, the heat transfer coefficient is predicted by Eq. C3 within an error less than $10 \%$ for gap widths smaller than $l_{f}^{s, \star} / 10=80 \mathrm{~nm}$. 


\section{2. p-polarization}

Similar approximations can be done to compute the contribution of frustrated total internal reflection in $\mathrm{p}$ polarization. Thus the transmission factor writes:

$$
A_{s}(\omega, \gamma, l, T) \simeq \frac{1}{1+\frac{\left(\gamma k_{0} l\right)^{2}}{I m\left[r_{p}\right]^{2}}}
$$

and

$$
\operatorname{Im}\left[r_{31}^{p}\right]=\frac{2 \varepsilon_{r} \gamma \sqrt{\varepsilon_{r}-1-\gamma^{2}}}{\varepsilon_{r}^{2} \gamma^{2}+\varepsilon_{r}-1-\gamma^{2}}
$$

The integration over the parallel wave-vectors gives:

$$
\begin{aligned}
& h_{f}^{p}(\omega, l, T)=h^{0}(\omega) \frac{\varepsilon_{r}-1}{2} \times \\
& \frac{\varepsilon_{r}^{2}}{a_{1}\left(1-\varepsilon_{r}^{2}\right)^{2}} \frac{1}{x_{m}-x_{p}} \times \\
& \left.\left\{\left(1-x_{p}\right) \ln \left(1-\frac{R}{x p}\right)-\left(1-x_{m}\right) \ln \left(1-\frac{R}{x m}\right)\right)\right\}
\end{aligned}
$$

where:

$$
\begin{aligned}
& a_{1}=\left(\frac{k_{0} l}{2}\right)^{2}\left(\varepsilon_{r}-1\right) \\
& x_{m}=-\frac{1}{\varepsilon_{r}^{2}-1}+\frac{\varepsilon_{r}^{2}}{2 a_{1}\left(\varepsilon_{r}^{2}-1\right)^{2}}-\frac{\varepsilon_{r}^{2}}{a_{1}} \frac{\sqrt{1+4 a_{1}\left(1-\varepsilon_{r}^{2}\right)}}{2\left(\varepsilon_{r}^{2}-1\right)^{2}} \\
& x_{p}=-\frac{1}{\varepsilon_{r}^{2}-1}+\frac{\varepsilon_{r}^{2}}{2 a_{1}\left(\varepsilon_{r}^{2}-1\right)^{2}}+\frac{\varepsilon_{r}^{2}}{a_{1}} \frac{\sqrt{1+4 a_{1}\left(1-\varepsilon_{r}^{2}\right)}}{2\left(\varepsilon_{r}^{2}-1\right)^{2}} \\
& R=\frac{n_{r}^{2}-1}{\varepsilon_{r}-1}
\end{aligned}
$$

The previous equations are the most general results concerning the contribution of p-polarized frustrated total internal reflections. In the asymptotic limits where $l \rightarrow 0$ and within the approximation $R \simeq 1$ the closedform expression simplifies as:

$$
\begin{aligned}
& h_{f}^{p}(\omega, l, T) \simeq h^{0}(\omega) \frac{n_{r}^{2}-1}{2} \times \\
& \left\{1+\left(\frac{k_{0} l}{2}\right)^{2}\left(\varepsilon_{r}-1\right) \varepsilon_{r}^{2} \ln \left[\left(\frac{k_{0} l}{2}\right)^{2}\left(\varepsilon_{r}-1\right) \varepsilon_{r}^{2}\right]\right\}
\end{aligned}
$$

The heat transfer coefficient reaches its saturation value when the second term in the bracket becomes negligible i.e. when gap widths $l$ are smaller than $l_{f}^{p, \star}=$
$\frac{\lambda_{T}}{\pi} \frac{1}{\varepsilon_{r}\left(\omega_{T}\right) \sqrt{\varepsilon_{r}\left(\omega_{T}\right)-1}}$. Compared to the characteristic gap width $l_{f}^{s, \star}$ in s-polarization the characteristic gap width in p-polarization is divided by a factor $\varepsilon_{r}\left(\omega_{T}\right) \simeq 10$ which explain why the p-polarization contribution reaches the asymptotic limit at extremely small gaps. At the room temperature Eq. (C5) predicts with an accuracy better than $10 \%$ the heat transfer coefficient for gap widths smaller than $l \leq l_{f}^{p, \star} / 10=8 \mathrm{~nm}$.

${ }^{1}$ E. G. Cravalho, C. L. Tien and R. P. caren, J. Heat Transfer 89, 351 (1967).

${ }^{2}$ Rytov, S. M., Y. A. Kravtsov, and V. I. Tatarskii, Principles of Statistical Radiophyics (Springer, New York,1989), Vol. 3.

${ }^{3}$ D. Polder and M. Van Hove, Phys. Rev. B 4, 3303 (1971).

${ }^{4}$ M.L. Levin, V. G. Polevoy and S. M. Rytov, Zh. Eksp. Teor. Fiz. 79, 2087 (1980) [Sov. Phys. JETP 52, 1054 (1980)]

${ }^{5}$ J. J. Loomis and H. J. Maris, Phys. Rev. B 50, 18517 (1994).

${ }^{6}$ J. B. Pendry, J. Phys.: Condens. 11, 6621-6633 (1999).

${ }^{7}$ A.I. Volokitin and B.N.J. Persson, Phys. Rev. B 63, 205404 (2001).

${ }^{8}$ J-P. Mulet, K. Joulain, R. Carminati and J-J. Greffet, Microscale Thermophysical Engineering 6, 209 (2002).

${ }^{9}$ S.Basu and Z. Zhang, Journ. Appl. Phys. 105, 093535 (2009).

${ }^{10}$ A.I. Volokitin and B.N.J. Persson, Rev. Mod. Phys. 79, 1291 (2007).

${ }^{11}$ K. Joulain, J.-P. Mulet, F. Marquier, R. Carminati, J.-J. Greffet, Surf. Sci. Rep. 57, 59-112 (2005).

${ }^{12}$ J.P. Mulet, K. Joulain, R. Carminati and J.J. Greffet, Appl. Phys. Lett. 78, 2931-2933 (2001).

${ }^{13}$ S. Shen, A. Narayanaswamy, G. Chen, Nano Lett. 9, 2009-2013 (2009)

${ }^{14}$ A. Narayanaswamy,S. Shen and G. Chen, Phys. Rev. B 78, 115303 (2008).

${ }^{15}$ E. Rousseau, A. Siria, G. Jourdan, S. Volz, F. Comin, J. Chevrier and J-J. Greffet, Nature Photonics 3, 514-517 (2009)

${ }^{16}$ S.-A. Biehs, E. Rousseau and J.-J. Greffet, Phys. Rev. Lett. 105, 234301 (2010).

${ }^{17}$ P. Ben-Abdallah and K. Joulain, Phys. Rev. B 82, 121419 (2010).

${ }^{18}$ S. Basu and Z. M. Zhang, Jour. of Appl. Phys. 105, 093535 (2009).

${ }^{19}$ X J Wang, S Basu, and Z M Zhang, Journal of Physics D: Applied Physics 42, 245403 (2009).

${ }^{20}$ C.J. Fu and Z.M. Zhang, Int. Jour. Heat Mass Transfer 49, 1703 (2006).

${ }^{21}$ E.D. Palik, Handbook of optical constants of solids, Volumes 1-4, Academic Press, New York

${ }^{22}$ F. Marquier, K.Joulain, J-P. Mulet, R. Carminati, J-J. Greffet and Y. Chen, Phys. Rev. B 69, 155412 (2004).

${ }^{23}$ M. Abramowitz and I. A. Stegun (ed.) Handbook of Mathematical Functions, National Bureau of Standards, 1964; reprinted Dover Publications, 1965.

${ }^{24}$ E. Rousseau, M. Laroche, J-J Greffet, Appl. Phys. Lett. 95, 231913 (2009). 